\title{
ARCHIVALISCHE MISZELLEN ÜBER O. BAUER
}

Auf eine Einführung in die unten veröffentlichten archivalischen Miszellen kann verzichtet werden: sie sprechen für sich selbst und wo immer eine Erläuterung angebracht erschien, wurde sie in einer Fussnote gegeben.

Bericht der Wiener Polizeidirektion vom i 2.III. 1918.

BAUER OtTo, Information.

Er lebt in günstigen finanziellen Verhältnissen und war für das Jahr I 9 I 2 für ein Einkommen von 4000 bis $4400 \mathrm{~K}$, für das Jahr I9I 4 für ein solches von 24.000 bis 26.000 Kronen und für das Jahr I9 Is für ein Einkommen von I9.000 bis $20.000 \mathrm{~K}$ besteuert. Ausserdem entrichtete er im Jahre I9I4 an Rentensteuer $247 \mathrm{~K}$ I3 h, die einem zu $4 \%$ verzinsten Kapital von $308.900 \mathrm{~K}$ entsprachen.

(MK-KM, 1918, 39, Nr. 15993.)

II

Qualifikationsliste des Dr. jur. OTто BAuER, Reserve-Kadettfeldwebel im Infanterieregimente Friedrich VIII., König von Dänemark, Nr. 75 .

Geboren: 5.IX.188 I zu Wien.

Heimatsquständig : nach Pollerskirchen, Bezirk Deutschbrod in Böhmen. Religion: israelitisch.

Sobn eines Fabrikanten.

Benebmen: dienstliches: Gegen Vorgesetzte sehr gehorsam und willig; gegen Gleichgestellte freundlich und zuvorkommend; gegen Untergebene - entschieden, consequent, angemessen strenge, daher wohlwollend. Versteht Manneszucht zu erhalten, wirkt auf die Mannschaft gut ein, genießt deren Vertrauen und Anhänglichkeit. 
außerdienstliches: Gegen Höhere: achtungsvoll und bescheiden; gegen Gleichgestellte freundlich und zuvorkommend; gegen Niedere angemessen.

Charakter-Eigenschaften: gut entwickelter Charakter, offen, vertrauenswürdig, sehr strebsam...

IIa

Vormerkblatt für die Qualifikationsbeschreibung für die Zeit vom 21.X.19I7 bis 31. August I9I8.

Leutnant i.d. Res. Dr. Otto Bauer.

Zum Leutnant bef. r.I.I909

Militär-Verdienstkreuz III. Kl. mit den Schwertern, Dez. 1917.

Im Krieg: 29.VII.-5.IX. Zugskommandant;

6.IX.-23.XI. Kompagniekomm.

Kampf nordöstlich Krakau 16.-23.XI. I9I4. 23.XI.1914-1.IX.1917 Kriegsgefangenschaft. 21.X.1917-31.X.1917 Kriegsministerium, Abt. Io KW/ W.G.X.

\section{III}

K.u.k. Reservespital in Brüx, E.Nr. s50 res.

Protokollarische Einvernabme des aus Rußland rückgekehrten Kriegsinvaliden. ad K.-M.-Erl., Abt. Io/Kgf, Nr. 10.465 vom 20. Mai I916.

Charge: Leutnant i. d. Res.

Name: Dr. Otto Bauer

Truppenkörper: I.R. 75

Unterabteilung: 9. Feldkompanie.

I Vorgänge, die zur Gefangennabme fübrten: Feind griff am 23.XI.I914 bei Bobin die von mir kommandierte Kompanie an und gelangte auf ıoo $\times$ Distanz. Ich entschloß mich zum Sturm. Der Sturm mißlang. Eigene Mannschaft lief zu dem eigenen Schützengraben zurück. Feind unternahm Gegensturm. Da ich bei dem von mir befohlenen Sturm meiner Mannschaft weit vorangeeilt war, mußte ich, als unser Sturm mißlang, hinter meiner Mannschaft her zum eigenen Graben zurücklaufen. Dabei wurde ich von den Russen, die jetzt ihrerseits stürmten, eingeholt, zu Boden geworfen und entwaffnet. 
2. Wabrnehmungen während der Gefangenscbaft binsichtlich der Bebandlung der Kgfn. und nach Aufzeichnungen und Erinnerungen über das Verbalten eigener staatsfeindlich gesinnter Heeresangehöriger: Im letzten Halbjahr wurde die Verpflegung der Krgsgef. in Sibirien wesentlich verschlechtert. - Im Gefangenenlager Berezowka, Transbaikalien, befanden sich am 20.VII. noch Invalide, die schon im Februar 1917 zum Austausch bestimmt wurden. Infolge der Verzögerung ihres Austausches gehen viele von ihnen, besonders die Tuberkulösen, zugrunde, die bei rechtzeitigem Austausch hätten gerettet werden können.

3. Abnabme von Geld und Wertgegenständen: Bei der Gefangennahme wurde mir nichts abgenommen. Erst bei dem Austausch vor dem Abtransport von Petersburg wurden mir im Spital Nr. 246 am 3i.VIII. abgenommen: I Uhr, I Rasierapparat, I verschlossener Holzkoffer mit Uniform- und Wäschestücken, I Lagerdecke.

4. Bebandlung der eigenen und feindl. Verwundeten und Kranken, erlittene oder selbstgesebene Misshandlung von Kriegsgefangenen: Bei meiner Gefangennahme wurde ich von der russ. Mannschaft mit den Gewehrkolben geschlagen, sodaß ich einige Tage nur mit großen Schmerzen gehen konnte. - Über Behandlung eigener Verwundeten und Kranken habe ich keine eigenen Wahrnehmungen.

5. Die Art, in welcher die Flucht ausgeführt wurde:

6. Erbalt von Wertpostsendungen und Beteilung von Hilfsaktionen (z.B. Besuche von Rote Kreuz-Scbwestern Österreich-Ungarns oder Deutschlands, Besucbe von Funktionären des Roten Kreuzes neutraler Staaten: Im Jahre I9 I kamen Wertpostsendungen in Berezowka und Troizkosawsko [?] fast nie an. Wenn Pakete ankamen, waren sie ihres Inhalts beraubt. Seit 1916 ist die Postzustellung etwas besser geworden. Auch die Liebesgaben des Roten Kreuzes kommen in Berezowka jetzt regelmäßig an; sie werden auf die Mannschaft durch österr.ung. Ärzte verteilt.

7. Besondere Angaben: Im July I 9 I 7 befahl das russ. Kriegsministerium meine Transferierung nach Petersburg. Ich wurde unter Bewachung eines russ. Soldaten von Berezowka nach Petersburg gebracht. Dort wurde mir ein Zimmer in der Wohnung eines russ. Militärarztes zugewiesen und ich erhielt die Erlaubnis, Besuche einiger mir persönlich befreundeter Herren zu empfangen, zu Studien-Zwecken eine öffentliche Bibliothek zu besuchen und in Begleitung eines Detektivs der „Kontr.-Razwjedka” (,Gegenspionage”) in der Stadt herumzugehen. Bei der Kontr.-Razwjedka mußte ich mich regelmässig melden.

Diesem besonderen Regime wurde ich ohne jedes Zutun meinerseits auf Intervention einiger mir befreundeter Russen unterworfen, 
die früher in Wien und in der Schweiz gelebt hatten, nach der Revolution nach Petersburg zurückgekehrt waren und dort jetzt großen Einfluß haben. Meine Transferierung nach Petersburg wurde auch von den russischen Zeitungen gemeldet; mein Name ist nämlich in Rußland bekannt, da einige meiner Bücher in russischer Übersetzung erschienen sind.

In Petersburg hatte ich einmal Gelegenheit, die russischen Minister Skobelew und Zeretelli zu sprechen. Skobelew kannte ich von Wien aus, wo er an der technischen Hochschule studiert hat; er stellte mich Zeretelli vor. Mein Gespräch mit den beiden Ministern hatte heftige Angriffe der russ. nationalistischen Presse gegen die Regierung zur Folge; sie warf den Ministern vor, daß sie sich „mit österreichischen Offizieren verbinden”.

Mein Aufenthalt in Petersburg dauerte nur einen Monat. Nach 4 Wochen wurde ich nämlich vor eine ärztliche Kommission gestellt, die mich zur Internierung in ein neutrales Land bestimmte. Mir wurde gesagt, die ärztliche Untersuchung sei auf Verlangen der österreichisch-ungarischen Regierung erfolgt, das k.u.k. Ministerium des Äußern habe das russische Rote Kreuz ersucht, meinen Gesundheitszustand festzustellen und mich im Bedarfsfalle in einem neutralen Lande internieren zu lassen. Wiener Verwandte teilen mir mit, daß das k.u.k. Ministerium tatsächlich auf diplomatischem Wege für mich interveniert habe; doch glaube ich, daß die russ. Regierung diesen Anlaß nur benützt hat, um sich meiner zu entledigen, weil ihr mein Aufenthalt in Petersburg infolge der Preßangst [?] unbequem war und sie mich aus Rücksicht auf meine Petersburger Freunde doch nicht in ein Gefangenenlager zurückbringen lassen wollte.

Brüx, am is. September I917.

Dr Otto Bauer, Lt. (m.p.)

[Am Rand ärztlicher Vermerk:] Apicat.. rechts, Herzneurose ohne erhebliche Funktionsstörung

IV

Der Vertreter des k.u.k. Ministeriums des Äußern beim k.u.k. Oberkommando, Baden, am 4. Oktober 1917. - Nr. 23289.

Siener Exzellenz dem Herrn Minister des k.u.k. Hauses und des Äußern Ottokar Graf Czernin.

Ich beehre mich, E. E. anverwahrt Abschrift eines Berichtes des Leiters der k.u.k. Kontrollstelle Sassnitz über die politischen und 
militärischen Verhältnisse der Ukraina, welcher interessante Daten über die Entwicklung der Lage daselbst enthält, zur hochgeneigten Kenntnisnahme zu unterbreiten.

\section{ad Nr. 23.289}

Ukrainische Angelegenbeiten.

Nach Aussagen der verschiedenen, hier durchkommenden Austauschinvaliden, insbesondere des Lts. Dr. Otto Bauer des IR. 75 ergänzen sich die von Exzellenz Szeptycki ${ }^{1}$ seinerzeit gegebenen Nachrichten zum folgenden Gesamtbild der gegenwärtigen Lage in der Ukraina. (Lt. Dr. Bauer hat durch Vermittlung ihm bekannter Mitglieder des Arbeiter- und Soldatenrates den letzten Monat seiner Gefangenschaft in Petersburg im Zustande blosser Konfinierung verbracht. In dem Bekanntenkreis, in dem er in dieser Zeit verkehrte, befanden sich zahlreiche politische Persönlichkeiten, als deren bedeutendste Lt. Dr. Bauer den Schriftsteller Martow ${ }^{2}$ nennt.)

Die Macht, die die Kiewer Rada über die Ukraina ausübt, ist einerseits eine politische, andererseits eine militärische. [...]

[Folgt ein ausführlicher Bericht über die politische, soziale und militärische Situation in der Ukraine. $]^{3}$

\section{V}

Kaiserlich Deutsche Botschaft in Wien. Nr. 26. Vertraulich!

Seiner Exzellenz dem Reichskanzler Herrn Grafen von Hertling, Wien, den 24. Januar $1918 .{ }^{4}$

Die Ausstandsbewegung liegt nach den heutigen Meldungen in den letzten Zügen. Die Blätter suchen die überwundene Gefahr nachträglich zu vertuschen und zu verkleinern und polemisieren gegen reichsdeutsche Zeitungen wegen übertriebener Schilderungen. Sie schämen sich der Schwäche des Staates und sie haben Grund dazu. Kann man sich vorstellen, daß die Regierung nicht einmal den Mut gefunden hat,

${ }^{1}$ Es handelt sich um den gr. kath. Metropoliten von Lemberg Andreas Gr. Szeptyckyj, der I9I5 von den Russen nach Russland verschleppt und erst Ende I9I 7 via Stockholm nach Österreich zurückkehren konnte.)

2 Im Original: Makow.

${ }^{3}$ Im Wiener Kriegsarchiv ist auch der archivalische Nachlaß der „Kontrollstelle Sassnitz” vorhanden. Leider wurden vom letzten Leiter der öst. Spionage Ronge viele Akten dieser Kontrollstelle vernichtet, und sogar ihre Inhaltsangabe im Protokoll mit einer Rasierklinge ausgeschnitten; dies geschah auch mit der Aussage O. Bauers.

4 Mit freundlicher Zustimmung von Herrn Legationsrat Dr. Ullich, Politisches Archiv des Auswärtigen Amtes, Bonn abgedruckt. Nr. A 3843, S. E $57054 \mathrm{I}$ f. 
in der kleinen Stadt Baden, dem Sitz des Hauptquartiers, eine größere Arbeiterversammlung zu verbieten.

Der Ausstand kam spontan von unten ohne jede Vorbereitung. Wieder einmal hat es sich gezeigt, daß solche Bewegungen ohne Organisation nicht lebensfähig sind. Die radikale Richtung, die unter der Arbeiterschaft die Oberhand gewonnen, hatte wohl Hetzer, aber keine organisierenden Führer. Der schlimmste Hetzer war ein Offizier, namens Bauer, der aus russischer Gefangenschaft heimgekehrt ist und mit Trotzky persönliche Verbindung gehabt haben soll. Er gilt geradezu als Abgesandter Trotzkys und hat die Arbeiter aufgefordert, sich nicht auf halbe Maßregeln zu beschränken, sondern „Feuer in die Häuser" zu werfen. Da er ein begabter Mensch sein soll, sieht man in ihm den zukünftigen Leiter einer radikalen Sozialdemokratie. Die Regierung hat es nicht gewagt, diesen gefährlichen Aufwiegler zu fassen. Unser General Franke erzählte mir, man wolle ihn nach Konstantinopel kommandieren, um ihn unschädlich zu machen. Mir erscheint es recht zweifelhaft, ob er dort unschädlich wäre; ich habe daher die Aufmerksamkeit des Generals von Cramon auf den Burschen gelenkt, der sich seiner annehmen will.

Die Massen haben ihre Macht und die Ohnmacht der Regierung gefühlt, sie haben grollend, weil sie nach ihrer Ansicht nicht genug erreicht haben, die Arbeit wieder aufnehmen müssen. Tatsächlich aber ging die Nachgiebigkeit der Regierung gegenüber den Arbeitern weit. Wie ich aus guter Quelle erfahre, ist sogar die verlangte Begnadigung Friedrich Adlers, des Mörders des Grafen Stürgkh, zwar nicht zugestanden, aber under der Hand für später in Aussicht gestellt.

Die Arbeiterschaft scheint sich für die Zukunft besser vorbereiten zu wollen. Ansätze zur Organisation der radikalen Richtung sind erkennbar. [...]

\section{VI}

ÖSTERREICHISCHE SPITZELBERICHTE ÜBER O. BAUER.

a) Passus aus einem Privatbriefe ddo. Stockholm, den 26. Jänner I 9 I 8 des Delegierten für Schweden der öst. Zentraleinkaufsgesellschaft (VEZEG), Dr. Aurel Richter an den k.u.k. Hof- und Ministerialvizesekretär im k.u.k. Ministerium des Äußern, Dr. Edwin Baron Versbach:

Hiesige gut unterrichtete Kreise teilen mir mit, daß die Unruhen in Österreich [gemeint ist der österreichische Januarstreik] von einem gewissen Otto Bauer in Scene gesetzt und angeführt worden seien. 
Derselbe war bereits vor dem Kriege österreichischer Staatsbürger, Sozialdemokrat, und machte gelegentlich der Zimmerwald-Bewegung die Bekanntschaft mit Trotzki. Sobald nun die Bolschewiki zur Macht kamen, verfügten sie die Freilassung des in der Zwischenzeit in russische Kriegsgefangenschaft gekommenen Bauer. Derselbe bekam direkte Unterrichtsstunden bei Herrn Trotzki und wurde hierauf nach Österreich entsendet. In Österreich angelangt, hat er es sofort verstanden, eine großzügige Propaganda für die Bolschewisten-Theorien zu entfalten. - Ich weiß nicht, ob der Betreffende sich noch auf freiem Fuße befindet; sollte dies aber der Fall sein, so müßte man gegen einen solchen gefährlichen Zustand sofort energische Gegenmaßregeln ergreifen.

Pro domo: Die Sache ist überholt. Bauer war bekanntlich im Kr. Min. angestellt, ist dermalen bei der „Arbeiterzeitung”. (MK-KM, 1918, 32, Nr. 13029.)

b) Vertreter d. Min.d.Äuß. beim AOK, Baden I 2.II. I9I8, Nr. 2672 I. Streng gebeim.

Wie mir das k.u.k. Armeeoberkommando mitteilt, hat General von Cramon nachstehende Zuschrift an Baron Arz gerichtet:

Verttauensleute der deutschen Obersten Heeresleitung melden: .....2) In Rußland gefangen gehaltene Österreicher tschechischer und polnischer Nationalität sind von dem ebenfalls dort interniert gewesenen Wiener Revolutionär Bauer organisiert worden und stehen geschlossen hinter Lenin. Bauer selbst soll zurzeit wieder in Österreich sein....

Über Bauer ist hier folgendes Telegramm bekannt, das die Agentur Kosmos am 28. September I9I7 aus Paris an Russkoje Slowo gesandt hat: [folgt die Notiz aus Le Temps, die wir unter Nr. VIII bringen].

c) Evidenzbüro des AOK, Nr. 4950, - 4.IV.1918.

Laut einer anher gelangten Mitteilung soll der bekannte russische Spion Waclaw Rabinowics an der revolutionären Propaganda arbeiten. Er ist gegenwärtig oder war wenigstens vor kurzer Zeit in Odessa (hat Holland im Mai verlassen) und führt nun den Namen Neumann. Er steht in Deutschland mit Mayer, dem früheren Redakteur des „Vorwärts”, und in Österreich angeblich mit Ba uer und auch noch mit anderen Personen, deren Namen nicht bekannt sind, in Verbindung. In Holland besorgt die Korrespondenz ein gewisser Nieuwenhuis.....

$$
\text { (MK-KM, I918, 42, Nr. 17202.) }
$$


d) Nr. 27793, Streng vertraulich

Referat zu Evb. Nr. 5616 von 1918.

....e) In Österreich-Ungarn soll ein großer Aufstand der Czechen und Südslaven in Vorbereitung sein, gleiches gilt bezüglich eines General streiks in diesem Staate, dessen Leitung und Organisation in den Händen des Wiener Revolutionärs Bauer liegt.

\section{VII}

RUSSISCHE ZEITUNGSNACHRICHTEN ÜBER O. BAUER.

a) „Iswestija”, 5.VII.1917:

Otto Bauer.

In der Abteilung für internationale Beziehungen des Exekutivkomitees ist die Nachricht eingetroffen, daß der Kriegsminister es für möglich hielt, dem kriegsgefangenen Leutnant Otto Bauer den Aufenthalt in Petersburg zu bewilligen.

b) Protest der „aus der Gefangenschaft Geflohenen” in „Russkoje Slowo" vom 3.VIII.1917 ( 558 Soldaten und 3 Offiziere) -

über die in der Tagespresse erschienene Nachricht, daß der gefangene österreichische Sozialist Otto Bauer an der gemeinsamen Sitzung des Zentralkomitees des Arbeiter- und Soldatenrates teilgenommen haben $[$ soll $][\ldots . . .$.

c) „Nowoje Wremia”, 8.IX.ı 9 I7.

Der ausgetauschte Sozialdemokrat Otto Bauer.

Der bekannte österreichische Sozialdemokrat Otto Bau er, welcher bei den Sitzungen des Sowjets anwesend war, wurde mit 43 anderen österreichischen Offizieren als invalider Kriegsgefangener ausgetauscht, - wahrscheinlich, um im Ausland genauen Bericht über die russischen Zustände zu geben. Mitte August I917 wurde von General Solowjow befohlen, daß der Kriegsgefangene Otto Bauer als invalid konstatiert und mit der ersten Gruppe der Austauschgefangenen nach Österreich abgeschoben werde. Obwohl Otto Bauer als vollkommen gesund konstatiert wurde, mußte er auf Befehl ausgetauscht werden. Es wäre wichtig zu erfahren, warum man sich um einen deutschen 
Sozialisten so bekümmert und wer an seinem Abtransport aus Rußland ein solches Interesse besitzt.

(Nr. 55473, 17.X.1917, - für den Minister.) ${ }^{1}$

\section{VIII}

\section{O. Bauer und Gr. Czernin.}

a) Tel. des Gr. Hadik aus Stockholm, 19.X.1917, Nr. 637, Chiffre.

Wie mir Stockholmer Vertreter der „Arbeiterzeitung” mitteilt, hat hiesiger Sovietvertreter [Vorovskij] folgendes Telegramm erhalten: „I 8. Oktober. In der bürgerlichen Presse ist eine Depesche aus Paris veröffentlicht, worin die Agentie Cosmos meldet: Ot to Bauer, der nach Österreich freigelassen wurde, wurde vom Grafen Czernin empfangen und hatte mit ihm eine längere Unterredung über die innere Lage Rußlands. Bauer ist jetzt in der russischen Abteilung des Ministeriums. Er wird mit seinem Rat helfen, die Bestrebungen der bolschewistischen Propaganda zu fördern, um dadurch die Politik Kerenskis und überhaupt den Einfluß der Anhänger einer energischen Fortsetzung des Krieges zu schwächen.

Wir erbitten eine sofortige Erklärung."

Ich erlaube mir, Euer Exzellenz um eventuelle Information zu bitten, um danach meine Sprache einrichten zu können.

b) „Le Temps”, 10.X.1917, S. 2:

Autriche-Hongrie.

Internationaliste et agent autrichien.

On mande de Vienne que le leader austro-hongrois, socialiste docteur Otto Bauer vient d'arriver dans cette ville après avoir passé trois anneés de captivité en Russie. Avant la guerre, le docteur Otto Bauer était directeur de la revue hebdomadaire Der Kampf (la Bataille), où il défendait le socialisme international dans sa forme extrême. Au début de la guerre, il fut appelé sous les drapeaux et fait prisonnier dans une des premières batailles de Galicie, en novembre I914.

'Die Nachricht über die Befreiung O. Bauers hatte nachstehende „Amtshandlung” der Ministerial-Kommission in Wien zur Folge:

Presseabteilung beim Oberkommando in den Marken, Nr. 1065, IX/5438:

Telegramm an die Redaktion des Berliner Tagblatts, Lugano, 6. Sept.

Kriegsgefangener österreichischer Sozialist Otto Bauer wurde durch russischen Arbeitersoldatenrat Freiheit gesetzt heimgeschickt.

Ministerial-Kommission, I I.IX.:

Gegen die Veröffentlichung des einliegenden Telegrammes obwaltet kein Anstand. 
Après la revolution russe, le docteur Bauer fut libéré du camp où il avait été interné et admis dans l'intimité du conseil des ouvriers et soldats de Petrograd, où il joua un rôle important en faisant du pacifisme dans l'intérêt des puissances centrales. Le gouvernement provisoire donna l'ordre d'échanger le docteur Bauer contre un autre prisonnier d'égale valeur.

Il est à remarquer que dans les vingt-quatre heures qui suivirent sa rentrée à Vienne, cet internationaliste militant fut reçu au ministère des affaires étrangères austro-hongrois par le comte Czernin, qui eut une longue conference avec lui sur la situation en Russie.

On annonce de Vienne que le docteur Bauer a été attaché à la „section russe" du ministère des affaires étrangères austro-hongrois ${ }^{1}$, où il sera, en quelque sorte, le conseil-expert, principalement sur la politique à suivre pour renforcer l'action des socialistes maximalistes en Russie et affaiblir ainsi la politique de M. Kerensky et la vigoureuse continuation de la guerre.

Die Mitteilung der Pariser Zeitung hat folgenden Aktenniederschlag gefunden:

$M K-K M$, 1917,8:

Nr. 3 1 25 Zensurweisung. Sozialist Otto Bauer, Verhandlungen mit Gr. Czernin und Anstellung desselben im Min. d. Äußern.

Nach mündlichen Verhandlungen des Min. d. Äußern mit der Red. der Arbeiterzeitung hat letztere in ihrer Nummer v. 21.X., S. 6 ein Dementi in Rede stehender Nachricht gebracht, auf das sich die Zensurweisung M.K.W. 3153 bezieht.

vid. Hh. Vertreter d.M.d.Âußern

pro domo: Die heutige $\mathrm{Nr}$ der Wiener Zeitung enthält bereits ein Dementi dieser Nachricht. 22.X.

Telegramm, 21.X.1917

Zeitungsnachrichten über angebliche Verhandlungen des Sozialisten Otto Bauer mit Grafen Czernin und Anstellung Bauer's im Ministerium des Äußern zu unterdrücken. - Ministrialkommission im Kriegsministerium, 3 I 25

Nr. 3 I 33 , nachträglich 23.X.

Im Nachhange zur Zensurweisung Z. 3 r25 vom 20. Oktober betreffend Otto Bauer wird eröffnet, daß Nachdruck des im Morgenblatte der Wiener Arbeiter Zeitung vom 2 I. Oktober veröffentlichten Dementis „Französische Presselügen über Otto Bauer” gestattet ist. Redaktion zu verständigen.

1 Eine Abteilung dieses Namens gab es nicht. 
c) „Arbeiter-Zeitung”, 21.X.1917, S. 6:

Französiscbe Presselügen über Otto Bauer.

Eine Pariser Lügenfabrik hat russischen Zeitungen die Nachricht zugeschickt, Otto Bauer sei nach seiner Rückkehr aus russischer Kriegsgefangenschaft ,vom Grafen Czernin empfangen worden und werde jetzt im Ministerium des Äußern zu politischen Zwecken, nämlich zur Förderung der Agitation der Bolschewiki verwendet”'. Diese Nachricht ist vom ersten bis zum letzten Wort eine dreiste Erfindung. Genosse Bauer hat den Grafen Czernin nie gesehen und er hat zum Ministerium des Äußern selbstverständlich keinerlei Beziehungen. ${ }^{1}$

1 Die Indices und die Protokolle des Ministeriums des Äußern enthalten keincrlei schriftliche Aufzeichnungen über eine Unterredung O. Bauers mit Gr. Czernin. 